\title{
Practical Phase Identification by Convergent Beam Electron Diffraction
}

\author{
JOHN MANSFIELD \\ North Campus Electron Microbeam Analysis Laboratory, University of Michigan, Ann Arbor, Michigan 48109-2143
}

\author{
KEY WORDS Analytical electron microscopy, Fingerprinting, CBED, Microanalysis
}

\begin{abstract}
The purpose of this article is to present a practical guide to the identification of phases in the analytical electron microscope with the aid of convergent beam electron diffraction. There is included a step-by-step approach to phase analysis, from the possible choices of the form of the specimen through how to explore reciprocal space in order to perform a full phase identification, either by symmetry analysis or by simple comparison of a pattern observed in the microscope with a previously recorded pattern (fingerprinting). There is a strong emphasis on practical hints and useful shortcuts.
\end{abstract}

\section{INTRODUCTION}

Although convergent beam electron diffraction (CBED) has a wide variety of applications, it is most frequently applied to the microanalysis of materials, since it is the single most powerful technique for the identification of crystalline phases in the analytical electron microscope (AEM). It is the only technique that can be used to identify the point and space group of individual submicron crystals. When this information is supplemented by chemical data from X-ray energy-dispersive (XED) or electron energy loss (EEL) spectra, the phase in question may be uniquely identified. There are a large number of papers in the literature that describe various aspects of CBED-based phase analysis and its application. The majority of examples of application tend to be in the general field of metallurgy, although, obviously, in no way does this imply that the technique is only suited to metallurgical samples; it may be applied to any crystalline specimen of reasonable perfection. Although well documented, CBED phase analysis techniques still have a certain amount of mystery associated with them; practitioners are prone to exaggerate their simplicity and novices are usually somewhat overwhelmed by the wealth of information contained in the actual patterns and assume that their complexity necessarily means that they are difficult to analyse. The mystery is compounded by the notion that one needs to know a good deal of group theory in order to analyse the patterns that one recrods. This is simply not true: an understanding of the mathematical aspects of group theory is not required; however, a knowledge of simple symmetry principles is required.

Another common misconception regarding CBED is that it must always be performed by using the smallest probe available in the microscope. This would normally involve using the scanning transmission (STEM) or nanoprobe (implying a probe of nanometer dimensions) mode of the microscope (the actual name of the mode depends upon the manufacturer of the instrument). It is not always necessary to resort to such extreme measures. The choice of probe size will be discussed further when we consider the choice of operating conditions of the microscope. A final preliminary comment regarding the application of CBED to phase analysis: it is apparent that there are people who conceive of CBED as a stand-alone analytical technique. While it is true that CBED alone may identify point and space groups, the technique gives little or no elemental information, and therefore for complete analysis it is absolutely necessary to use XEDS and EELS to determine the elemental composition of the phase(s) under investigation.

Although it is assumed throughout the remainder of this article that the reader is a newcomer to CBED, and thus many of the aspects, often considered trivial, will be discussed in detail, a basic knowledge of crystallography and symmetry will be assumed and much reference will be made to the other articles that are collected together in this publication as they form a cohesive set.

\section{PRACTICAL ASPECTS OF PHASE ANALYSIS Preamble}

We approach CBED phase analysis starting with the very basics. The chart in Figure 1 outlines a basic approach to phase characterization via CBED. The chart shows, of necessity, an idealized procedure as the exact process will vary because the lengths to which one has to go to perform a full analysis of an unknown phase depend upon a variety of criteria. Two basic steps are missing from the flow chart: specimen preparation procedures optimized for CBED and the basic learning and acclimatization process which is necessary when first starting to perform CBED analysis. These two steps, and the literature survey for information on the particular phase system of interest, can really be taken

\footnotetext{
Received February 24, 1989; accepted in revised form March 24, 1989

Address reprint requests to John Mansfield, North Campus Electron Microbeam Analysis Laboratory, University of Michigan, 2455 Hayward, Ann Arbor MI 48109-2143.
} 


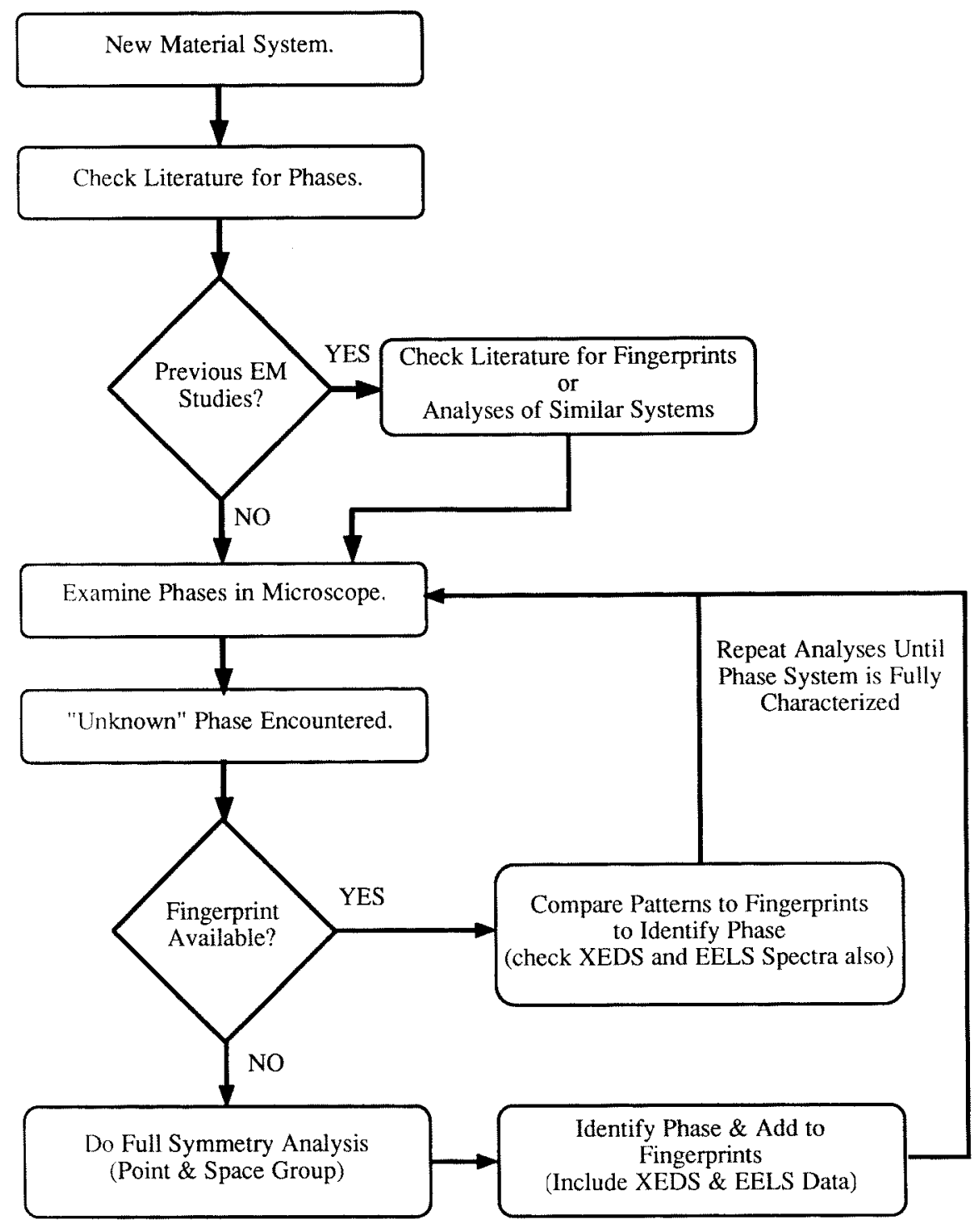

Fig. 1. Schematic diagram of the recommended steps to be followed when performing phase analysis by CBED.

in any order and how an individual proceeds is a matter of personal preference.

One of the first questions that should be asked when considering phase analysis by CBED is: How much is already known about this chemical system? There will always be some background information for the materials under examination. If there have been previous electron microscope examinations of the phase system in question, then the whole procedure is likely to be more straightforward. Even if the sample is a total unknown, say a meteorite or geological sample, there is usually some indication of the possible composition. Generally there will be a good deal known about the chemical composition of the starting material and also its thermomechanical history; hence a search of the relevant literature should yield some phase diagram and crystallographic information. Before beginning to examine the specimen one should be reasonably familiar with the compositions and structures of the phases that are usually found in the chemical system in question. However, microanalysis of materials is frequently employed to determine the reasons for the failure of an in-service component, for example, an aircraft part or a microelectronics device, and it is worth noting that materials that have been mass produced in industry are still likely to contain undesirable impurity elements and phases, in spite of the considerable developments in materials processing that have occurred over the last few decades. Therefore, it is quite possible to come across a hitherto 
undiscovered phase, particularly in complex systems containing three, four, five, or more elements and a few random impurities (Graham, 1984; Rackham and Steeds, 1980).

\section{Specimen Considerations}

When it has been determined which phases are most likely to be encountered, the temptation to try immediately to examine the material in the microscope should be avoided, since it would be wise to first consider the best way to prepare the sample so that the diffraction analysis will be as simple as possible. Although there is not always more than one possible procedure for preparing TEM specimens, it is always worth considering the alternatives, particularly if the process of finding and recording diffraction patterns is simplified. CBED is usually most straightforward if performed on particles that are well separated from one another on a carbon-supporting film (e.g., powders scatterd on carbon-coated copper grids or carbon extraction replicas of metallic samples). The particles can be easily viewed from the diffraction mode of the microscope by defocussing the second condenser lens and observing the shadow image of the particle in the brightfield disc of the diffraction pattern. Obviously not all materials lend themselves to such convenient preparation. Thin foil samples, whether chemically or electrochemically polished or ion-milled, require a little more care in characterization. The shadow image approach can be used for thin-foil samples; however, it is more difficult to recognize crystallites that are equi-axed particularly if they are surrounded by similar features that change in shape as the specimen is tilted. Before spending valuable time slowly tilting a second phase embedded in a matrix toward what appears to be a major zone axis, tilting the stage over large angles is recommended, to check that the particle of interest does not become occluded by the phases around it or the edge of the holder. (This problem also applies to specimens on carbon films, since the grid bars frequently obstruct the pattern).

\section{Learning CBED Techniques}

When initially performing CBED experiments, one should practice on a specimen that is not important, since it is possible to contaminate or damage samples in the learning process. The samples containing the actual unknown phases should be stored safely for a couple of microscope sessions while CBED is practiced on something simple; a thin foil of steel, aluminium, or a piece of silicon is ideal. A good practical tutorial article-"How to Get a Convergent Beam Electron Diffraction Pattern and How to Recognize What You See", by Alwyn Eades (1985)_is recommended. This is a step-by-step guide to finding a zone axis from a randomly oriented direction and analysing the symmetry of the axis. Practice on a test sample should continue until the individual is confident that he is unlikely to damage more valuable specimens.

The initial analyses should avoid trying to solve too many problems simultaneously. The CBED patterns displayed in Figure 2 are fairly typical of those presented in the literature, although the author has avoided using a silicon zone axis pattern (ZAP) as a typical example and has chosen patterns that formed part of actual phase identification studies. Both these patterns are high-camera-length high-quality ZAPs. However, it is fairly obvious that if one randomly selects a particle or grain in the electron microscope, focuses a small probe on it, and selects the diffraction mode, the resulting pattern will not look anything like those Figure 2. Whereas the reason for this is obvious to most people who have used selected area diffraction-the crystal is obviously not oriented along a low-order crystallographic direction-the solution to the problem may not be considered straightforward. It is necessary to make use of the goniometer and a tilting stage to find a major crystallographic direction. The commonest type of tilting holder found in AEMs is the double-tilting stage. These are usually preferred because the secondary tilt usually causes comparatively little movement of the specimen area of interest. In addition a double-tilt stage is relatively simple to fabricate in beryllium, which ensures a "low background" in XEDS. Such holders are not, however, the holders of choice for CBED. A tilt-rotation holder not only allows access to a larger volume of reciprocal space but makes "steering around" in reciprocal space somewhat easier, once one has become used to the large amount of specimen movement that occurs when rotating an area of the specimen that is far from the center of rotation.

Navigation in reciprocal space can be very easy, particularly when the specimen under study yields good Kikuchi lines. Generally the Kikuchi information seen in CBED patterns is much clearer than in SAD patterns since the diffraction is averaged over a much smaller volume of crystal-which is generally a good approximation to a perfect single crystal. It is worth considering at this point the optimum probe size for CBED. It is frequently not necessary to use the smallest probe that the microscope is capable of producing, particularly when you are in the process of tilting the crystal to a prominent zone axis. As an example, say the size of the phase under examination is of the order of a few microns, then using a $5 \mathrm{~nm}$ probe is clearly overkill. A comparatively large probe will result in brighter patterns that are easier to see on the microscope screen, although the Kikuchi detail generally will be less sharp for the reason discussed above. When a zone axis has been reached, then the probe size can be reduced so that the detail in the CBED disks is well defined and undistorted. If there is evidence of the presence of faulting or dislocations, it may be necessary to reduce the probe size and/or move the probe around the crystal to select an area that yields distortion-free patterns.

\section{Choosing Patterns for Maximum Information}

Having experimented with CBED, one will find that it is no great achievement to record a zone axis pattern with low symmetry. Not only do most crystals, even those with very-high-symmetry point groups, have a number of low-symmetry zone axes, but high-quality patterns from high-symmetry axes often degrade rapidly into a diffuse, unrecognizable mess. The reason for 


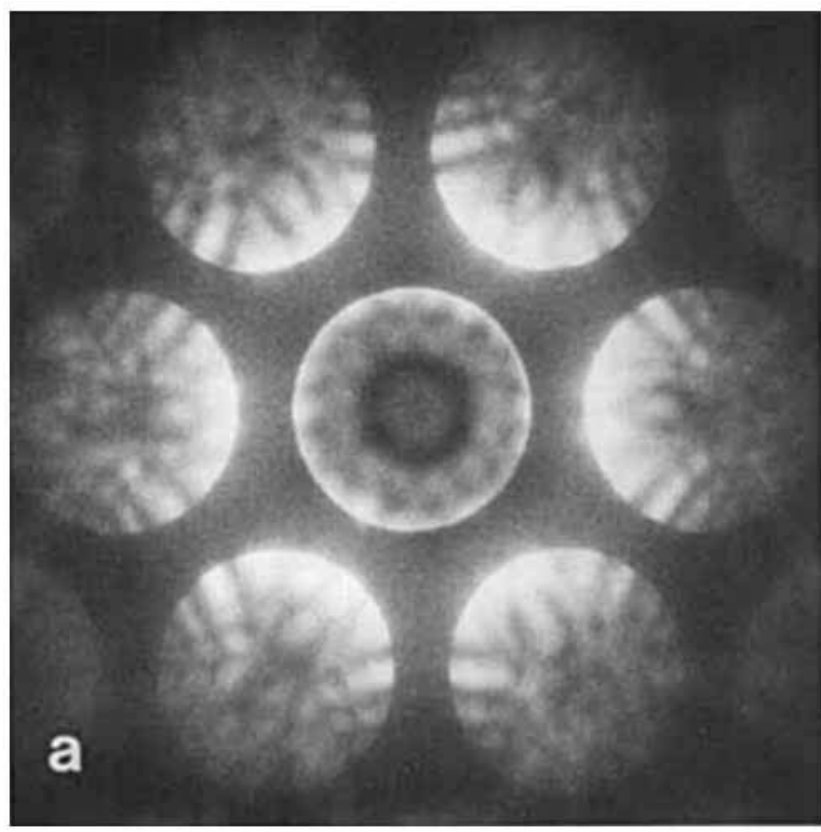

Fig. 2. Two high-camera-length zone axis patterns. Although by no means typical of those encountered every day in routine analysis, these were both recorded during actual research studies and not especially for this article. a: $A<111>$ pattern from a small particle of indium (body centered tetragonal, space group $14 / \mathrm{mmm}$ ) in an aluminium matrix. The specimen was not very thick and the disks are well separated; however, there are no HOLZ lines and this is probably

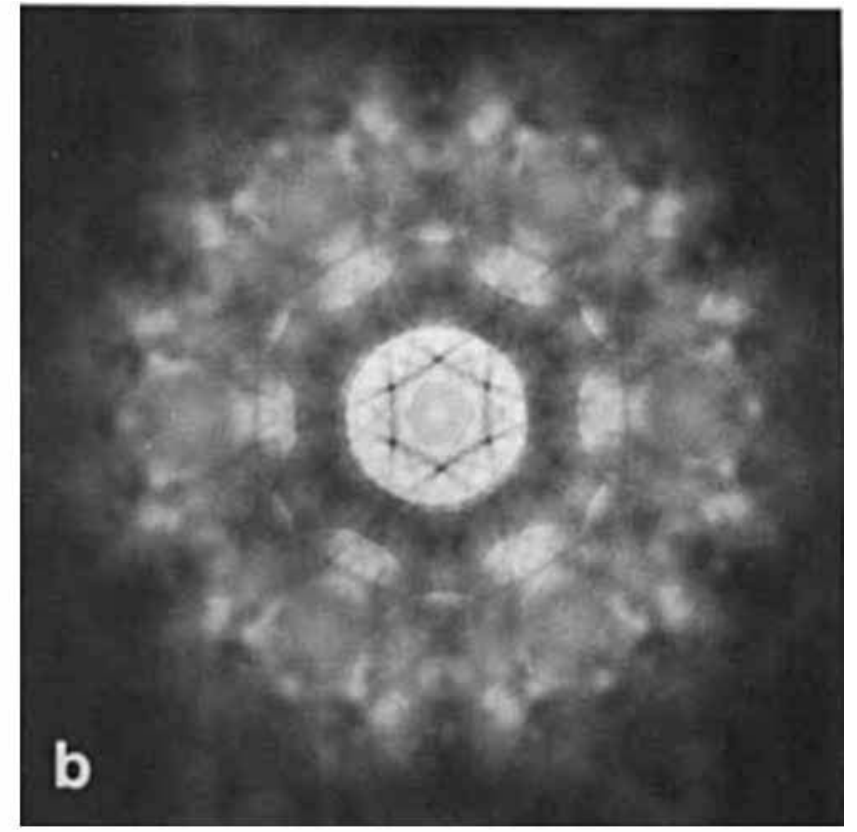

due to the fact that indium-based foils were very difficult to prepare defect free since the material was so soft. Pattern recorded at $80 \mathrm{kV}$ and reproduced courtesy Dave Van Aken. b: A [0001] zone axis from a Laves phase $\left(\mathrm{MN}_{2}\right.$, hexagonal, space group $\mathrm{P}_{3} / \mathrm{mmc}$ ) found in long-term creep-tested AISI 316 stainless steel. Pattern recorded at $100 \mathrm{kV}$ with no specimen cooling (from The Bristol Group, 1984).

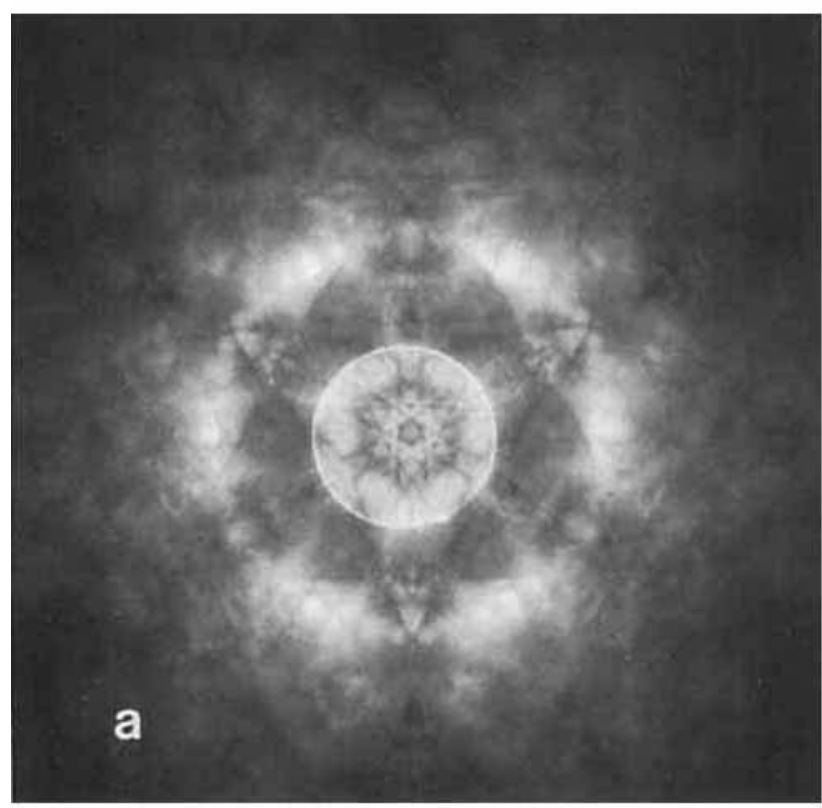

Fig. 3. Two high-camera-length ZAPs from $\mathrm{M}_{23} \mathrm{X}_{6}$ (face-centered cubic, space group $\mathrm{Fm} 3 \mathrm{~m}$ ) discovered in a 316 stainless steel. a: $<111>$ A large condenser aperture has been here and the discs overlap heavily. The $3 \mathrm{~m}$ symmetry of the pattern is very clear. b:

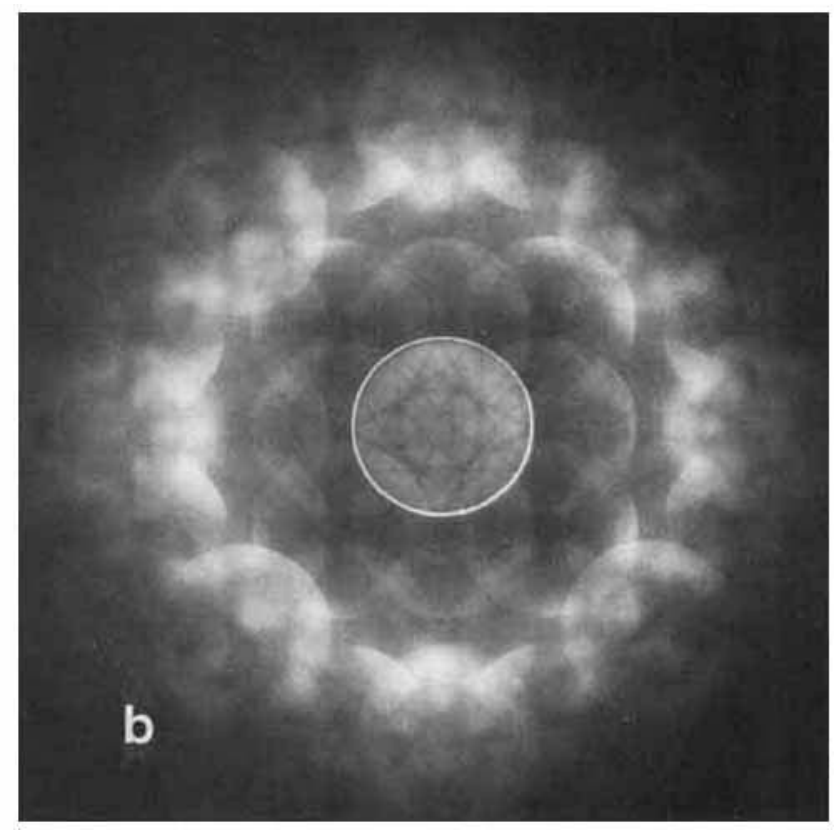

$<100>$ pattern. Again a large $\mathrm{C}_{2}$ aperture has led to overlapping discs but a clear delineation of the symmetry. From The Bristol Group (1984), recorded at $100 \mathrm{kV}$ with no specimen cooling. 
the degradation is that CBED is sensitive to a wide variety of effects that are usually not visible in SAD. Strain fields from dislocations and faults or surface contaminant films can seriously distort the symmetries of the patterns and point disorder quickly "smears-out" the high-angle scattering. Disorder may be introduced into a sample merely by examining it, as, even at 100 $\mathrm{kV}$, the electron beam has sufficient energy to damage a large number of materials, particularly those containing a large proportion of light elements. Nonetheless, it is preferable to search for and record high symmetry zone axis patterns if they occur in the crystal system of the studied phase, because they will yield the maximum amount of information regarding the crystal point group. High-symmetry axes are also much more distinctive than those with low symmetry and may be so recognizable that one or two alone may serve to identify a phase. This concept of fingerprinting is discussed in detail later. With a little practice, one can tilt a specimen quickly to a high-symmetry zone axis and close scrutiny of that one axis may allow identification of the point group (Steeds and Vincent, 1983).

When using selected area diffraction, it is usually neither necessary nor possible to centre perfectly the zone axis pattern that is being recorded; the $d$-spacing and angular information can be obtained from imperfectly aligned patterns. The same applies to CBED patterns. However, if the patterns are to be used for symmetry determination then it is important to have the electron beam well aligned with the crystallographic zone axis of interest. Although with a small amount of practice it is possible to quickly tilt/rotate small crystals to a zone axis, the final high-precision centering is best not done by mechanical means because of the backlash limitations of the goniometer and holder. Final centering is usually done by making small shifts of the second condenser aperture or by using the beam-tilt coils of the microscope. Such tilts should, however, be limited to a few milliradians as excessive amounts of tilt in this manner result in pattern distortion (Carpenter and Spence, 1984).

It may be necessary to record the pattern at different exposures to identify the point group. A very-longcamera-length ZAP may be used to determine the symmetry of the brightfield disc; an intermediate camera length is useful for comparing the symmetry of the zero layer with that of the brightfield disc; and a verylow-camera-length pattern may be used to examine the higher-order Laue zone (HOLZ) rings to determine the whole pattern symmetry. To reveal the internal darkfield symmetries and the relationships of pairs of hkl and $\overline{h k l}( \pm G)$ darkfield reflections, it is necessary to displace the $\mathrm{C} 2$ aperture and record high-cameralength patterns (see, for example, Tanaka, this issue; Tanaka and Terauchi, 1985).

\section{EXAMPLES OF PHASE ANALYSIS}

Now that we have considered how to record CBED patterns, let us examine a few examples of phase analysis. This section covers the extreme cases of phase analysis: 1) analysis by symmetry considerations, with a reminder of the possible problems in low-symmetry systems, and 2) fingerprinting. These two extremes encompass the full range of phase analysis by CBED.

\section{Symmetry Examples}

The micrographs in Figure 3 are patterns, recorded at $100 \mathrm{kV}$, of two major zone axes from the metallic carbide $\mathrm{M}_{23} \mathrm{X}_{6}$. Figure $3 \mathrm{a}$ is a high-camera-length pattern at the $\langle 111\rangle$ axis. Because the specimen was rather thick and because of the relatively large lattice parameter, the discs overlap and they are therefore are difficult to see. However, the pattern symmetry is clearly $3 \mathrm{~m}$. Figure $3 \mathrm{~b}$ is the $<100\rangle$ zone axis, again with overlapped dises, and it shows $4 \mathrm{~mm}$ symmetry, Examination of the pattern symmetry tables first published by Buxton et al. (1976), but reproduced elsewhere in this publication, reveals that the possible diffraction groups with a whole pattern and brightfield symmetry of $3 \mathrm{~m}$ are $3 \mathrm{~m}$ and $6_{\mathrm{R}} \mathrm{mm}_{\mathrm{R}}$, and for $4 \mathrm{~mm}$ the groups are $4 \mathrm{~mm}$ and $4 \mathrm{~mm} 1_{\mathrm{R}}$. Diffraction group $3 \mathrm{~m}$ has possible point groups of $3 \mathrm{~m}$ and $\overline{4} 3 \mathrm{~m}$ while $6_{R} \mathrm{~mm}_{\mathrm{R}}$ has point groups of $\overline{3} \mathrm{~m}$ and $\mathrm{m} 3 \mathrm{~m}$. For the fourfold axis the possibilities are $4 \mathrm{~mm}$ for diffraction group $4 \mathrm{~mm}$ and $4 / \mathrm{mmm}$ and $\mathrm{m} 3 \mathrm{~m}$ for $4 \mathrm{~mm}_{\mathrm{R}}$. The point group of this crystal is therefore $\mathrm{m} 3 \mathrm{~m}$, since this is the only group consistent with both orientations. Although the point group could have been determined from either pattern alone, by measurement of the ratio of the FOLZ ring radius to the spot spacing of the zero layer and observing how the network of FOLZ reflections map on to the zero layer, the method presented here provides an alternative technique to those usually quoted (Tanaka, this issue; Tanaka and Terauchi, 1985; Steeds, 1983 ), is based on purely symmetry arguments, and requires no measurement or indexing. It does, of course, require more tilting to obtain two ZAPs with different symmetries. However, when examining a new phase a considerable amount of tilting is necessary to ensure that the maximum information is extracted in one microscope session. This is particularly true if one wishes to identify the space group, since it may be necessary to check several orientations for the presence of dynamic absences (Buxton et al., 1976).

\section{ZAP Maps $\mathrm{M}_{23} \mathrm{X}_{6}$ and $\mathrm{CdTe}_{2} \mathrm{O}_{5}$}

In the process of tilting around reciprocal space to locate high-symmetry axes one will find many axes of relatively low symmetry, each of which may exhibit the highest symmetry hitherto observed, thus it is wise to record these patterns as they are discovered. In this manner one can construct reciprocal space maps that are usually very useful in later analyses. A typical ZAP map is shown in Figure 4. This map is recorded from the $\mathrm{M}_{23} \mathrm{X}_{6}$ phase discussed above and covers a standard stereographic triangle. Such a ZAP map is simple to construct since, for the high symmetry cubic structure, unique axes occur in 1/24th of the stereographic projection. Crystals with lower symmetries have ZAP maps that cover larger areas. An orthorhombic map, for example, covers one-quarter of the projection and a monoclinic map nearly half. Obviously construction of such maps from low-symmetry crystals would be more time consuming since it would generally not be possible, even given the wide range of angles available from 


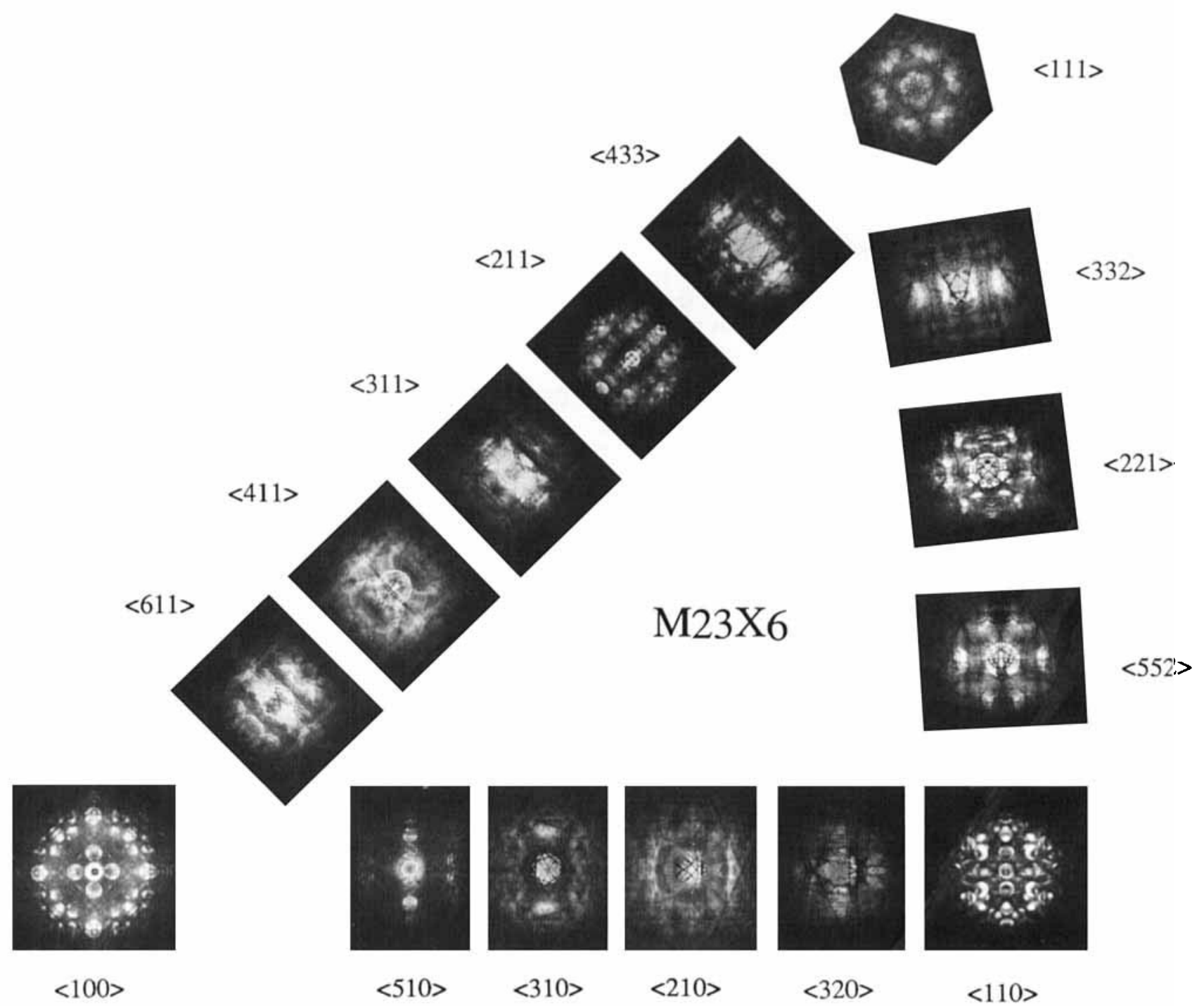

Fig. 4. Zone axis pattern map for the $\mathrm{M}_{23} \mathrm{X}_{6}$ phase featured in Figure 3. All patterns recorded at 100 $\mathrm{kV}$ with no specimen cooling employed. Map covers a standard stereographic triangle (from The Bristol Group, 1984)

a tilt-rotate holder, to complete a map of either an orthorhombic or a monoclinic cell from one particle.

To emphasize this point consider Figure 5, which is a calculated Kikuchi map for the monoclinic phase $\mathrm{CdTe}_{2} \mathrm{O}_{5}$ (space group $\mathrm{C} 2 / \mathrm{m}$ ). The real space zone axis pattern map has recently been recorded for this phase by Silva and Scozia (in press). Here it will serve to illustrate an extreme case which would probably require tilting around a large volume of reciprocal space before locating the major symmetry features of the phase. Let us assume that one is examining a particle of the phase in the microscope and the first major zone axis that is discovers is labelled \#1 in the figure (it is in fact [112]). Close examination in the microscope reveals that the pattern is very difficult to center and the symmetry appears to be one. After recording the pattern one would usually chose to tilt the pattern along one of the strongest Kikuchi bands and search for a higher symmetry ZAP. The next ZAP, \#2 ([314]), also appears to have symmetry of one and the next couple of axes are clearly very low order ([516] and [718]). After approximately $32^{\circ}$ total tilt axis \#3, ([101]), is located and it contains an obvious mirror, as indicated on the figure. Tilting along the mirror to find higher-symmetry axes would reveal that the mirror is the highest-symmetry operator along that Kikuchi band. After locating two or three more zone axes containing only a mirror, one would probably try and tilt $90^{\circ}$ to the mirror line to find a rotation axis. If such a tilt were possible, given the constraints of the specimen and holder, one would find that the ZAP $90^{\circ}$ away contains a simple twofold rotation axis and hence the crystal is monoclinic. 


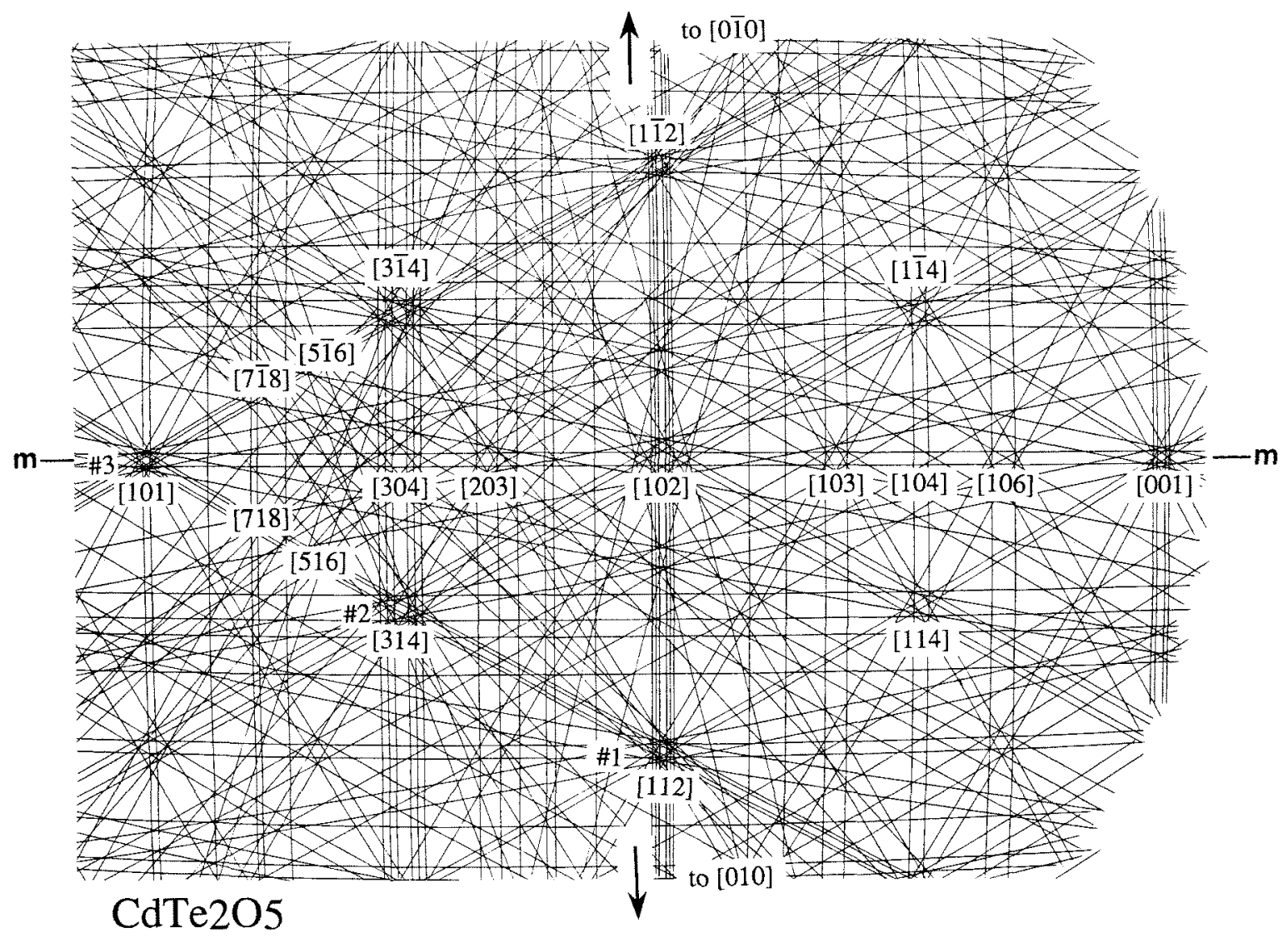

Fig. 5. Section of a $100 \mathrm{kV}$-calculated Kikuchi map for $\mathrm{CdTe}_{2} \mathrm{O}_{5}$, which has a monoclinic c-centered unit and space group $\mathrm{C} 2 / \mathrm{m}$. The figure as a whole only possesses a single mirror, which is labelled. Courtesy Silva and Scozia.

\section{Fingerprinting}

The Alternative Approach to Phase Analysis. The case above is obviously a nontrivial case for analysis and it is chosen to illustrate how much may be involved in the characterization. It also emphasizes the usefulness of constructing ZAP maps and storing highquality ZAPs from phases that are discovered, to use as "fingerprints" in the future. The human eye is very skilled at pattern recognition and CBED ZAPs are very distinctive, so although some ZAPs may appear very similar to one another, a close look usually allows the identification of unique features. As an example of this, Figure 6 shows two pairs of ZAPs that have similar features but are from different, although related phases (The Bristol Group, 1984). Figure 6a is composed of two $<111>$ ZAPs; $6 \mathrm{a}$ is from the austenitic matrix phase (gamma) of a nickel-based superalloy and $6 \mathrm{~b}$ is the strengthening phase (gamma') of the same alloy. Both were recorded at identical operating conditions. At first glance they are very similar; however, the gamma prime ZAP clearly has extra reflections which can be seen as low-intensity disks which partially overlap the direct disk. These are the 110 reflections that are allowed in the primitive cubic gamma prime but are forbidden in the face-centered cubic austenite. Figure 7 includes two ZAPs from a titaniumbased alloy with additions of vanadium (Mansfield et al., 1986). Both ZAPs appear to have approximately sixfold projection symmetry; however, only $7 \mathrm{a}$, the $<111\rangle$ axis from the cubic $\beta$-phase, has $6 \mathrm{~mm}$ projection symmetry, whereas $7 \mathrm{~b}$, the [1120] axis from the hexagonal alpha phase, actually only has $2 \mathrm{~mm}$ symmetry. An extensive analysis of alpha titanium employing several different approaches to CBED has been performed by Howe et al. (1986).

Practical Examples: $M_{23} B_{6}, M_{7} X_{3}$, and SiC. It is obvious that if phases can be identified by simple comparison of ZAPs, then the initial search of the literature should include a check for preexisting ZAPs and/or maps of phases that are likely to exist in the phase system under current study. There are several publications that have been put together expressly to provide examples of high-quality ZAPs from as wide a variety of phases as possible; these include Tanaka and Terauchi (1985), The Bristol Group (1984), and Mansfield et al. (1986). A good example of the application of fingerprinting was reported by Kruger et al. (1988). Figure $8 \mathrm{a}$ is a typical zone axis pattern recorded from particles observed in an Ni-Cr-Fe alloy; the phase was $\mathrm{M}_{23} \mathrm{X}_{6}$ and it was identified by direct comparison with the pattern in Figure $8 \mathrm{~b}$, which is from the published 

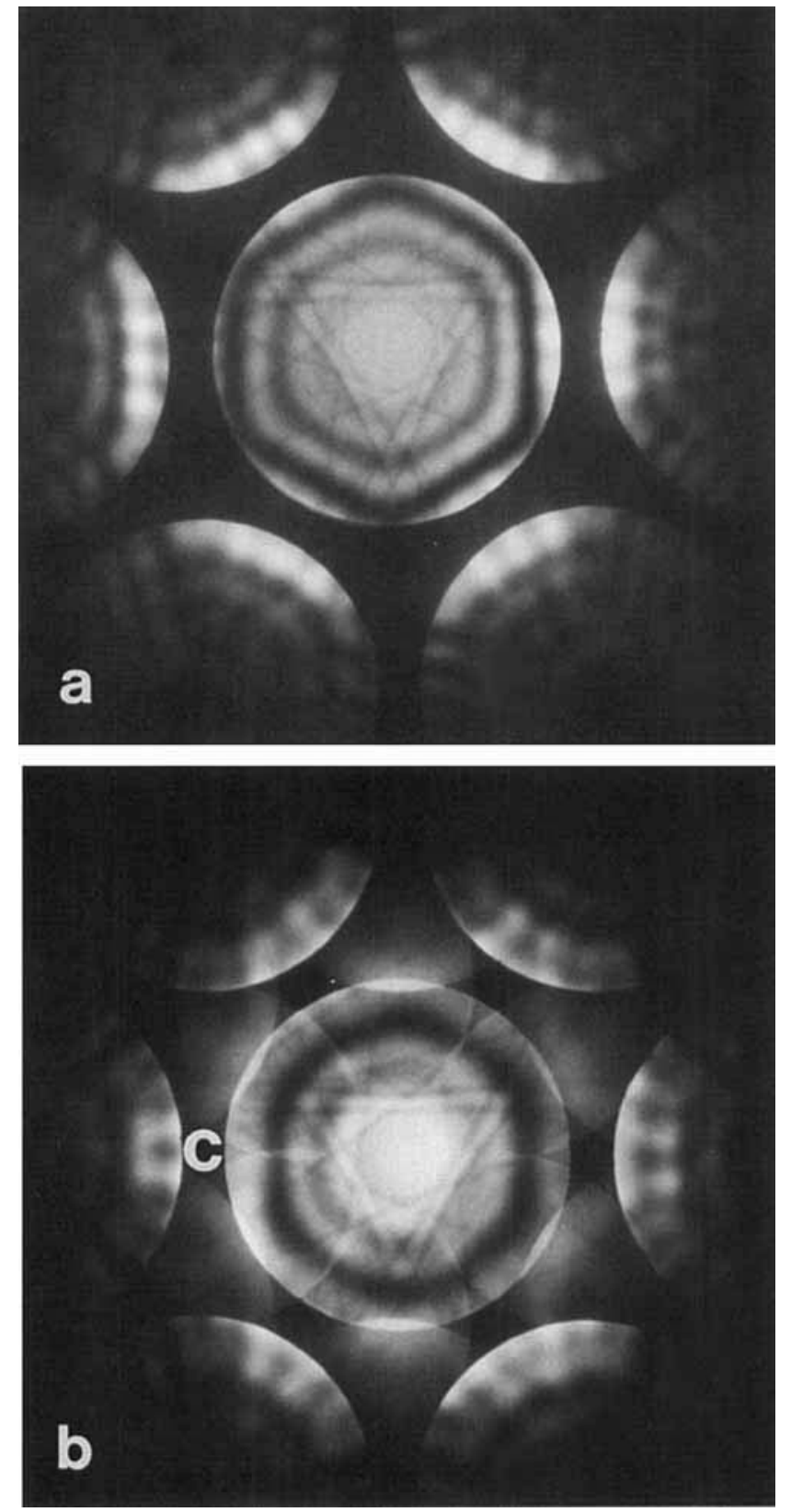

Fig. 6. <111> zone axis patterns recorded at identical operating conditions from the gamma austenite (a) and the gamma-prime strengthening phase (b) from a nickel-based superalloy. Although the fine details of the brightfield disc are similar and the darkfield disc spacing is nearly identical the presence of the 110 reflections (one of which is labbelled C) in the gamma-prime pattern allows them to be distinguished. Patterns recorded at $120 \mathrm{kV}$ and without specimen cooling.

collection Convergent Beam Electron Diffraction of Alloy phases (The Bristol Group, 1984). There is little if any detail in the discs; however, the intensity variations from disc to disc are so distinct that there is no difficulty in performing the comparison and identifying the phase. However, complete identification of the
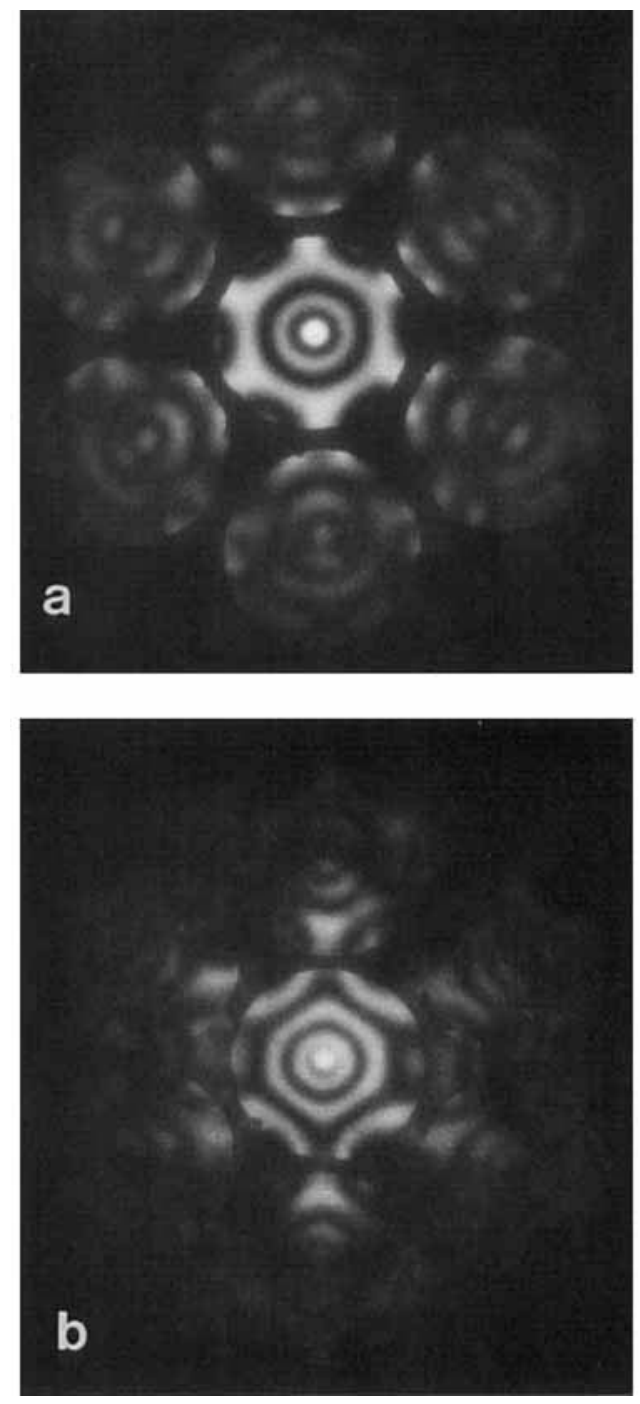

Fig. 7. Zone axis patterns from a titanium vanadium alloy. a: A $<111>$ axis from $\beta$-titanium which is a body-centered cubic phase with space group $\operatorname{Im} 3 \mathrm{~m}$. b: The [11 20$]$ axis of the hexagonal alpha titanium phase which has space group $\mathrm{P}_{3} / \mathrm{mmc}$. Although both ZAPs appear to have sixfold projection symmetry at first glance; only the $<111>$ pattern has $6 \mathrm{~mm}$; the $[11 \overline{2} 0]$ axis in fact only has $2 \mathrm{~mm}$ symmetry. Patterns recorded at $120 \mathrm{kV}$ at room temperature (from Mansfield et al., 1986).

phase required the use of XEDS and EELS. The XED spectrum contained a good deal of nickel, and since nickel does not readily form carbides, it was necessary to resort to EELS to determine the light element content. The $\mathrm{X}$ component was found to be entirely boron; hence the phase was actually $\mathrm{M}_{23} \mathrm{~B}_{6}$.

Another fingerprinting example from the same alloy system is illustrated in Figure 9. The orthorhombic/ pseudohexagonal phase $\mathbf{M}_{7} \mathbf{X}_{3}$ is common in steels and alloys and is frequently heavily faulted. The $1.2 \mathrm{~nm} \mathrm{c}$ axis spacing means that the CBED discs overlap heavily unless a very small aperture is used. Unfortunately this makes symmetry details difficult to see. The [010] ZAP in Figure 9a was identified by comparison 

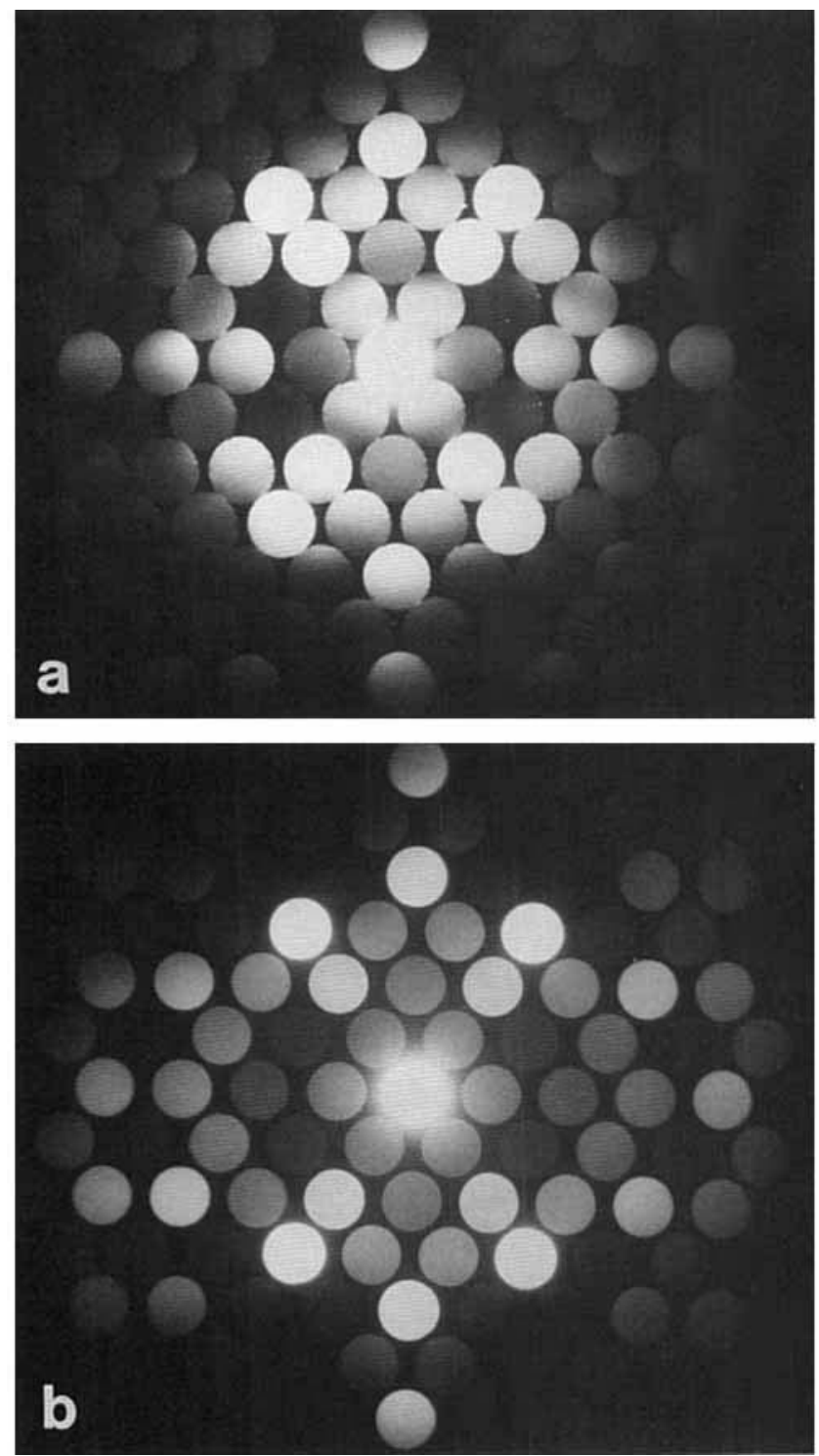

Fig. 8. Example of CBED fingerprinting in a nickel-chromiumiron alloy, a: $\mathrm{A}<110>$ zone axis pattern recorded from a particle located in the Ni-Cr-Fe alloy. The specimen is rather thin but the intensity variations in the discs form a distinctive pattern (Kruger et al., 1988). It was identified, in the microscope, by comparison with the pattern in $\mathbf{b}$ which is a $<110>$ ZAP from an $\mathbf{M}_{23} \mathrm{X}_{6}$ particle in 316 steel and is from Convergent Beam Electron Diffraction of Alloy Phases (The Bristol Group, 1984). Patterns recorded at $100 \mathrm{kV}$.

with that in Figure $9 \mathrm{~b}$, which is from the alloy phases text (The Bristol Group, 1984). Although they appear different, the ratios of the principal spacings in the zero layer are the same and the FOLZ ring diameters were found to be in the same proportion to the zero-layer spacings. This datum together with the XED spectra and the morphology of the particles was sufficient to identify the phase. Note that the ZAP recorded from the $\mathrm{Ni}-\mathrm{Cr}-\mathrm{Fe}$ has more clearly visible dark bars in the horizontal odd-numbered reflections through the origin.
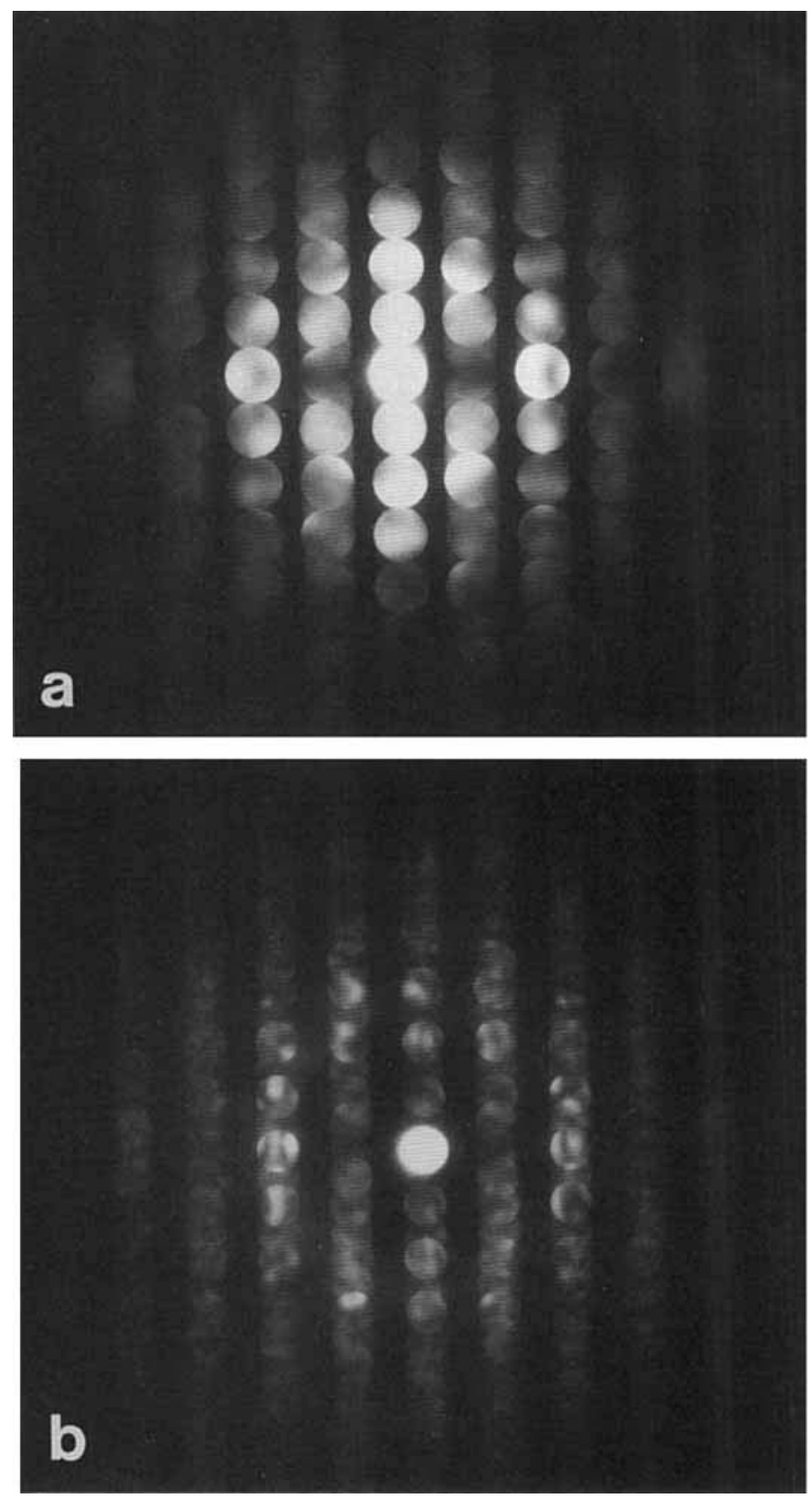

Fig. 9. A second example of CBED fingerprinting from the same alloy as in Figure 8. a: From the Ni-Cr-Fe alloy. b: From The Bristol Group (1984). Both patterns in this figure are at the [010] axis of the orthorhombic phase $\mathrm{M}_{7} \mathrm{X}_{3}$. They were both recorded at $100 \mathrm{kV}$ but from different-thickness crystal and $\mathrm{C}_{2}$ aperture sizes. Identification required the measurement of the disc spacing and the FOLZ ring diameter as well as examination of the XED spectrum and the precipitate morphology. The horizontal dark bars, most clearly visible in the top pattern, are due to the presence of both a horizontal screw axis and parallel glide plane.

These are dynamic absences due to the presence of both a parallel glide plane and a perpendicular $2_{1}$ screw axis at this zone axis (for further discussion of dynamic absences see Tanaka, this issue). The improved clarity is 

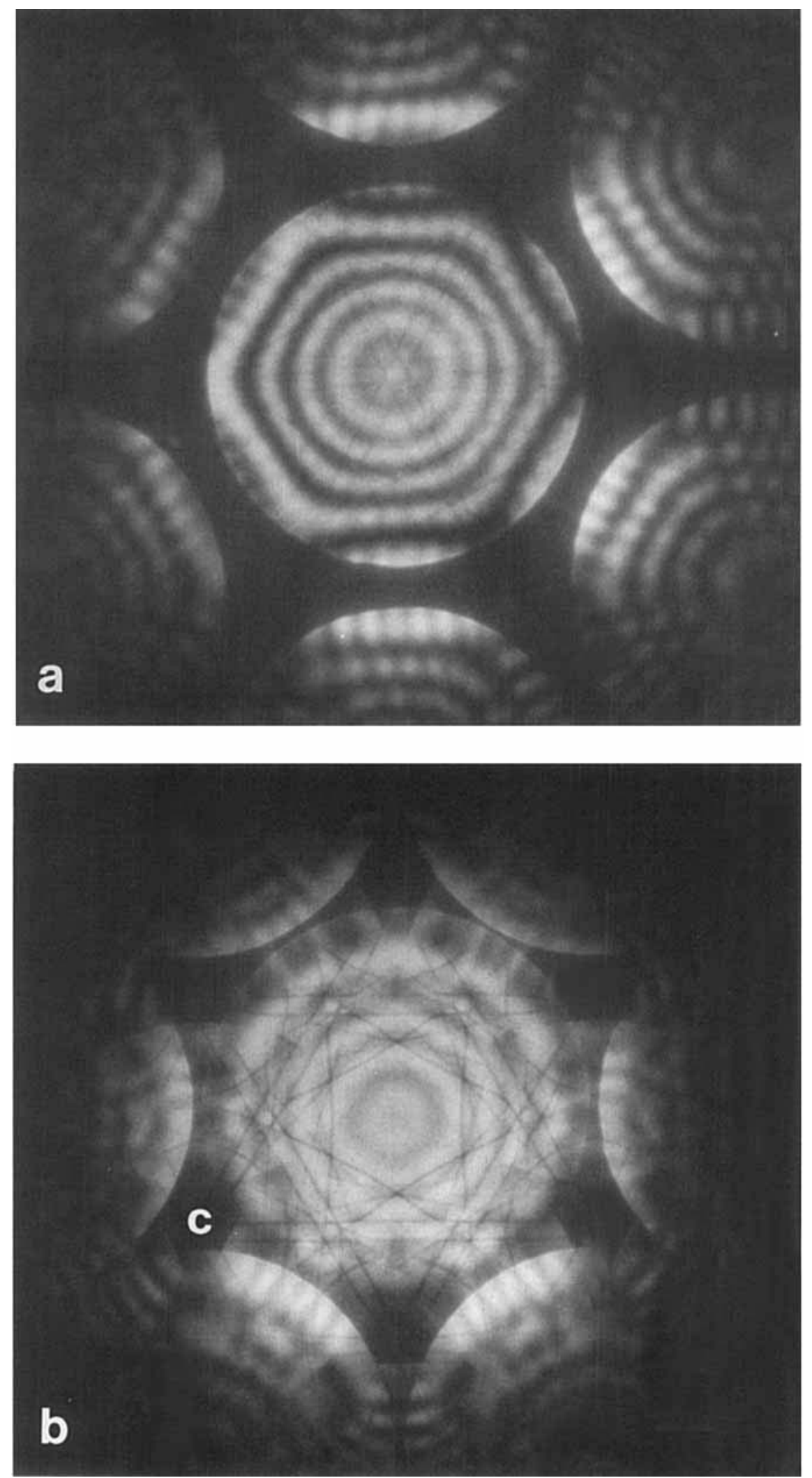

Fig 10. [0001] zone axis patterns from two polytypes of silicon carbide (space grup $\mathrm{P}_{3} \mathrm{mc}$ ). a: From $6 \mathrm{H}$ silicon carbide. b: The $4 \mathrm{H}$ polytype. Both are recorded at the same voltage $202.7 \mathrm{kV}$. Note the weak 1010 discs present in the $4 \mathrm{H}$ pattern (c labels the center of one of them) which overlap the zero layer disc; these reflections are forbidden in the $6 \mathrm{H}$ poltype. Patterns courtesy Hangas et al. (1986). 

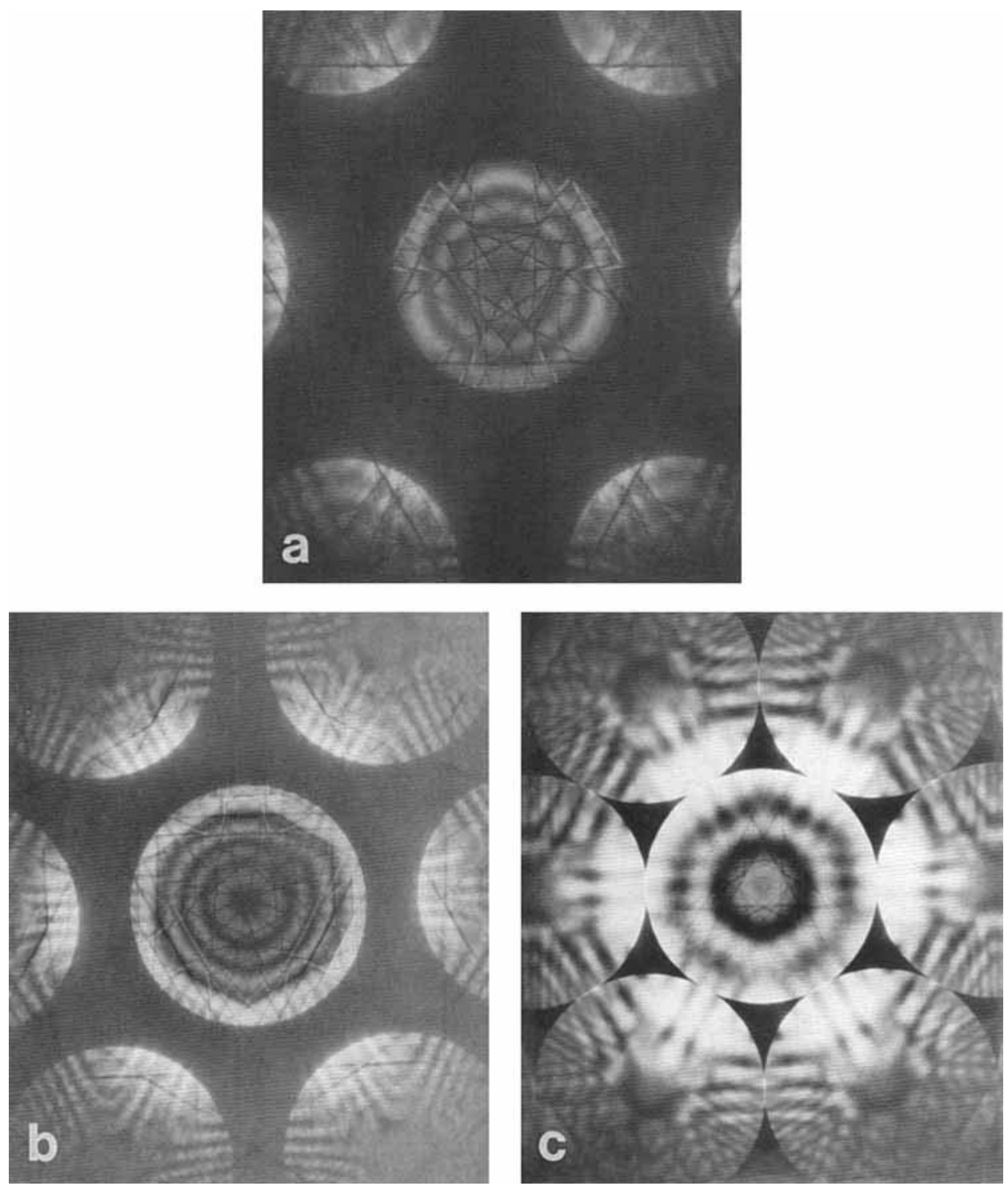

Fig. 11. $<111>$ Zone axis patterns recorded from pure silicon at three different accelerating voltages. a: $100 \mathrm{kv}$. b: $150 \mathrm{kv}$. c: $200 \mathrm{kv}$. Fingerprints from different voltages are therefore not comparable.

due to a specimen of different thickness and a different second-condenser aperture size from those used in the original fingerprint and this pattern forms a superior fingerprint for this axis of $\mathrm{M}_{7} \mathrm{X}_{3}$.

The two fingerprinting examples above were from relatively thin crystals, where the scattering is pseudokinematic. However, fingerprinting also has applications in thick crystals. An example where fingerprinting may be employed with strongly dynamic patterns is illustrated with the [0001] silicon carbide ZAPs shown in Figure 10 (Hangas et al., 1986). Figure 10a is from $6 \mathrm{H}$ silicon carbide and Figure $10 \mathrm{~b}$ is the $4 \mathrm{H}$ polytype. Both are recorded at the same voltage (approximately $200 \mathrm{kV}$ ) and are clearly distinguishable. Note that there are weak $10 \mathrm{I0}$ discs present in the $4 \mathrm{H}$ pattern which overlap the zero-layer disc. These reflections are forbidden in the $6 \mathrm{H}$ polytype. For each of the cases described, $\mathrm{M}_{23} \mathrm{X}_{6}, \mathrm{M}_{7} \mathrm{X}_{3}$, and the $\mathrm{SiC}$, the standard patterns (fingerprints) were recorded from material that was being examined in the course of a research program; it is not necessary to prepare special standard specimens for the establishment of fingerprints. In fact, any convergent-beam pattern which has been recorded at a well-characterized operating condition of the microscope and identified can be used subsequently as a fingerprint.

\section{DISCUSSION, CAUTIONS, AND CONCLUSIONS}

The examples presented above represent the extremes of phase analysis by CBED. The most funda- 

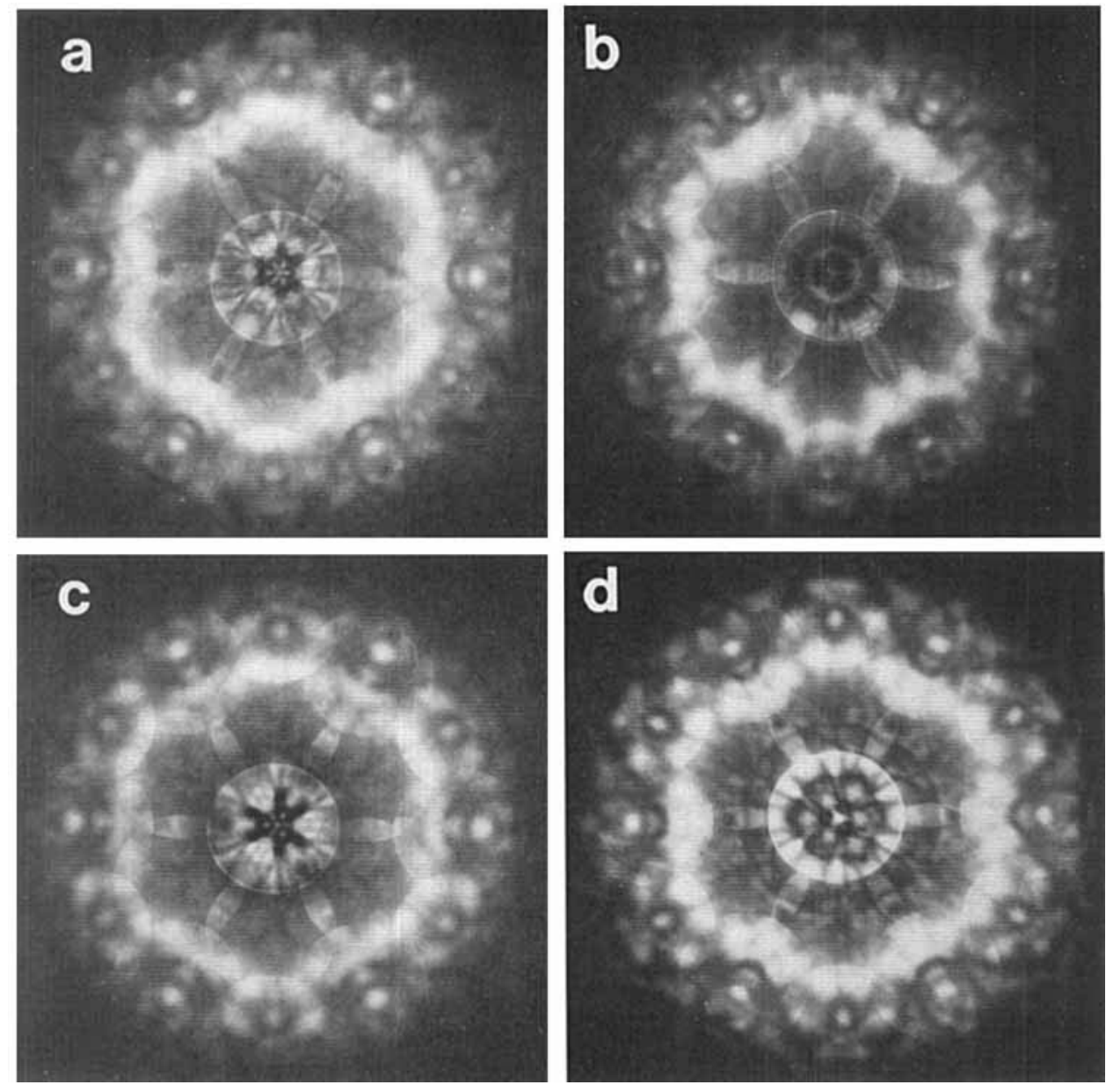

Fig. 12. <111> ZAP thickness sequence from a sample of $\mathrm{M}_{6} \mathrm{X}$ or eta-carbide. The patterns do not change in a recognizable way as a function of thickness. Recorded at $100 \mathrm{kV}$ (from The Bristol Group, 1984).

mental approach involves a full crystallographic analysis of the reciprocal lattice of the phase to identify the point and space group and lattice parameters. This method can prove quite time consuming. On the other hand, it can be seen that we have also discovered that the analysis can be completed in a few minutes if a preexisting fingerprint can be found. Although providing a collection of fingerprints has been the purpose of the publication of a number of the books and articles, it is generally not possible, or desirable, to perform CBED phase analysis with the sole intention of constructing sets of fingerprints. Rather it is preferable to establish the fingerprints as the characterization of a particular phase system progresses, occasionally collecting together fingerprints from related phases in periodic handbooks, where the accent is on the clarity of the diffraction patterns rather than how the phases relate to the properties of the material in which they are found.

In the studies that led to the creation of Convergent Beam Electron Diffraction of Alloy Phases (The Bristol Group, 1984) it became obvious that the process of fingerprinting could often be accelerated by the use of XEDS, as it is frequently possible that a particular phase may have a very well-defined and invariant XED spectrum. The phases were usually initially identified by CBED and their X-ray spectra were examined over a number different occurrences of the phase. In the case of phases in 316 steel and a nubmer of nickel-based superalloys there were a number of phases that did not have a fixed composition. However, the majority could be reliably identified by XED fingerprinting alone.

Although little mention has been made of the third major analytical technique in the TEM, EELS, it now appears that it also may have a future as a fingerprint technique. Because EELS is usually very sensitive to changes in thickness, it is usually considered of limited use as a fingerprint. However, Zaluzec (1987) has shown recently that the use of logarithmic digital filters may effectively remove the effects of thickness from EELS data and hence make it possible to compare spectra from different specimens. 
It is a major purpose of this article to illustrate the usefulness of CBED for fingerprinting. Nevertheless, there are a number of cautions that should be mentioned. Recently the validity of the technique has been questioned for reasons that are related to the question of recognition (Bird et al., 1987; Mansfield, 1987). The principle of fingerprinting relies upon the assumption that each convergent beam pattern is distinct and recognizably so. Few people disagree that each CBED pattern is distinct. The dissension would occur with the word recognizably. The most obvious variables that change the appearance of CBED ZAPs are voltage and thickness. Most early CBED studies were performed on microscopes that had a maximum operating voltage of $120 \mathrm{kV}$, and Convergent Beam Electron Diffraction of Alloy Phases was for the most part based on data that were recorded at $100 \mathrm{kV}$ because it was necessary for several researchers to be able to compare data since they were involved in studies of similar materials. Recent developments by manufacturers of AEMs has led to the use of operating voltages of up to $400 \mathrm{kV}$. This presents an even greater problem since fingerprints obtained at $400 \mathrm{kV}$ are significantly different from those obtained at $100 \mathrm{kV}$. This is illustrated, although for a narrower voltage range, in Figure 11, which is a series of long-camera-length silicon $<111>$ ZAPs recorded at three different voltages. It should also be noted that there are some limitations on using the higher intermediate voltages since the dynamical scattering is reduced and much thicker material has to be used to yield equivalent symmetry detail. It is therefore preferable to perform CBED phase analysis at a standard voltage if possible. Although goodquality CBED ZAPs have been recorded from austenitic stainless steel up to $200 \mathrm{kV}$ (Mansfield, 1987), 100 $\mathrm{kV}$, which is available on virtually all TEMs, is a more reasonable choice.

Problems related to thickness are less readily solved since it is sometimes the case that the features within the zero layer dises of a pattern are very sensitive to thickness and do not change in any clear progression as the thickness is increased. Examples of this are certain axes of the Laves phase that was seen in Figure 2 and the so-called eta-carbide $\left(\mathrm{M}_{6} \mathrm{X}\right)$ that is frequently discovered in steel (Fig. 12). In such cases the only solution is to construct a fingerprint that consists of a set of patterns from different thicknesses and make a note that, in order to perform a comparison, the electron beam should be moved around on the unknown phase to sample different thicknesses to obtain a match.

There is still some feeling that high-energy electron diffraction is so sensitive that the presence of small numbers of defects, surface contaminants, and specimen thickness wedges make fingerprinting impossible (Bird et al., 1987). However, if used with care it is usually possible to avoid these detrimental effects For this reason it is really necessary to record micrographs on film in order to examine the very fine details to ensure that there are not subtle effects present that are not obvious on the viewing screen of the microscope.

\section{ACKNOWLEDGMENTS}

The author would like to thank Elisa Silva and Ricardo Scozia of the University of Chile, Santiago, Chile, for permission to use information from a forthcoming paper and Jon Hangas of The Scientific Research Laboratory of Ford Motor Company, Dearborn, Michigan, and Dave Van Aken of The Department of Materials Science and Engineering at the Unviersity of Michigan for allowing the use of some of their micrographs as examples.

\section{REFERENCES}

Bird, D.M., Eaglesham, D.J., Kaufman, M.J., and Mansfield, J.F. (1987) CBED Workshop Discussion Group. In: Analytical Electron Microscopy 1987. D.C. Joy, ed. San Francisco Press, San Francisco, p. 176.

The Bristol Group (1984) Convergent Beam Electron Diffraction of Alloy Phases, Bristol. Adam Hilger Ltd., U.K.

Buxton, B.F., Eades, J.A., Steeds, J.W., and Rackham, G.M. (1976) The symmetry of electron diffraction zone axis patterns. Proc. R Soc. Lond., A281:171

Carpenter, R.W., Spence, J.C.H. (1984) Applications of modern microdiffraction to materials science. J. Microsc., 136:165-178.

Eades, J.A. (1985) How to get a convergent beam electron diffraction pattern and how to recognize what you see. Norelco Reporter, $32(1 \mathrm{EM}): 22-37$.

Graham, R.J. (1984) An Investigation of the Microstructure of AuGeNi Contacts to InP. Ph.D Thesis, University of Bristol, Bristol, UK.

Hangas, J., Shinozaki, S., and Donlon, W.T. (1986) Lattice parameter measurements of SiC materials using CBED HOLZ lines. In: Proceedings of the 44th EMSA. G.W. Bailey, ed. San Francisco Press, San Francisco, pp. 464-465.

Howe, M., Sarikaya, M., and Gronsky, R. (1986) Space group analyses of thin precipitates by different convergent beam electron diffraction procedures. Acta Cryst., A42:368-380.

Kruger, R.M., Was, G.S., Mansfield, J.F., and Martin, J.R. (1988) An experimental and theoretical investigation of the intergranular precipitation of M7X3 and M23X6 in Ni-16Cr-9Fe-C-B. Acta Met. 12:3163-3176.

Mansfield, J.F., Graham, R.J., and Lin, Y.P. (1986) The Library of Convergent Beam Electron Diffraction, update no. 1. Norelco Reporter, 33(1EM):54-66.

Mansfield, J.F. (1987) Conventional convergent beam electron diffraction: Is fingerprinting a viable interpretive approach? In: Analytical Electron Microscopy 1987. D.C. Joy, ed. San Francisco Press, San Francisco, p. 138.

Rackham, G.M., and Steeds, J.W. (1980) Nickel, germanium, gold contacts to gallium phosphide. Inst. Phys. Conf. Ser., 52:157.

Silva, E., and Scozia, R. (1988) University of Chile, Santiago, Chile, manuscript in press.

Steeds, J.W. (1983) Electron crystallography. In: Quantitative Electron Microscopy, Proc. 25th SUSSP. Scottish Universities Summer School in Physics Publications, Edinburgh, Scotland. J.N. Chapman and A.J. Craven, eds. pp.49-96.

Steeds, J.W., and Vincent, R. (1983) Use of high-symmetry zone axes in electron diffraction in determining crystal point and space groups. J. Appl. Cryst., 16:317-324.

Tanaka, M., and Terauchi, M. (1985) Convergent Beam Electron Diffraction. JEOL Ltd., Tokyo, Japan.

Tanaka, M. (1989) Symmetry analysis. J. Electron Microse. Tech., 13:27-39.

Zaluzec, N.J. (1987) Spectral processing and quantitative analysis in electron energy loss spectroscopy using a digital filter technique. In: Analytical Electron Microscopy 1987. D.C. Joy, ed. San Francisco Press, San Francisco, p. 275. 